Volume 9, No.1.4, 2020

International Journal of Advanced Trends in Computer Science and Engineering

Available Online at http://www.warse.org/IJATCSE/static/pdf/file/ijatcse8091.42020.pdf

https://doi.org/10.30534/ijatcse/2020/8091.42020

\title{
Student Emotion Estimation Based on Facial Application in E-Learning during COVID-19 Pandemic
}

\author{
Nurbaity Sabri ${ }^{1}$, Nur Hamiza Musa ${ }^{2}$, Nur Nabilah Abu Mangshor ${ }^{3}$, \\ Shafaf Ibrahim ${ }^{4}$, Hazwa Hanim Mohamed Hamzah ${ }^{5}$ \\ ${ }^{1,2,3,4,5}$ Faculty of Computer and Mathematical Sciences, Universiti Teknologi MARA (UiTM) Melaka, Kampus \\ Jasin, 77300 Merlimau, Melaka, Malaysia, \\ nurbaity_sabri@uitm.edu.my, nurnabilah@uitm.edu.my, shafaf2429@uitm.edu.my, hazwa@uitm.edu.my
}

\begin{abstract}
The COVID-19 pandemic occurred in late 2019, and by the beginning of 2020 the entire education system has shifted from traditional teaching methods to online learning systems around the world. COVID-19 reinforces the need to explore online learning and learning opportunities. However, the ability of teachers to recognize and see how individual students engage in online learning is more challenging. Student emotions such as self-esteem, inspiration, dedication, and others that are assumed to be determinants of student success cannot be overlooked. The main objective of this research is to evaluate the emotion of student on e-learning during COVID-19 pandemic using a facial recognition application. This application able to interpretation of facial expressions into extracted emotional states. An image processing approach has been implements in 4 types of emotion which is happy, normal, sad and surprise. Next the image will go through the identifying of emotion type from the static frontal face image. It starts with image acquisition, grayscale conversion and contrast stretching for image pre-processing, Haar Cascade or also known as Viola-Jones technique for face detection, face model technique for eye and mouth localization, skin-color segmentation technique for image segmentation, and Grey-Level Co-Occurrence Matrix (GLCM) for feature extraction. The classification for emotion type is using SVM Regression. The accuracy percentage of emotion classification is calculated. The result showed that SVM Regression has a high accuracy rate of $99.16 \%$. A real-time application will be developed to identify human face emotion instead of static image for future work with additional of speech recognition exploration.
\end{abstract}

Key words : COVID-19 pandemic, e-learning, emotion, facial recognition, image processing.

\section{INTRODUCTION}

Today, the COVID-19 pandemic is pushing educational institutions, such as universities, to migrate quickly to distance and online learning. This pandemic has forced universities around the world to engage in online learning. A different and accessible learning solution, such as e-learning systems and mobile learning applications, is crucial. Online learning is not new to learners or distance learning. However, COVID-19 is reinvigorating the need to explore online learning and learning possibilities [1]. Variety of approaches has been implemented to resolve the spread of infection with COVID-19. Social distancing and lock-down initiatives have changed human behaviors, as the Internet has played a major role in promoting remote learning, e-teaching, online networking, gaming, video sharing, and more. The rise of digital technologies and learning management systems in teaching and assessment has contributed to an alternative for educators to be able to use information technology during the quarantine period [2].

Numerous latest technologies have been adopted by e-learning over the last decade to enhance the efficiency, efficiency and attractiveness of e-learning. Internet-based technologies are seeking to address existing educational needs, closing the gap between conventional education approaches and future developments in technology-combined education [3]. To this end, a number of advanced e-learning systems lack functionalities such as instructional interactive environments, customized features, and learning data monitoring and relevance feedback [4]. Emotions are a key component of effective learning and problem-solving, particularly when it comes to engaging with computer-based learning environments [5]. In this research, a medium to improve effectiveness of e-learning during COVID-19 pandemic has been developed. The aim to obtain an accuracy of student emotion estimation by implements the facial recognition has been done.

Human easily change their facial emotion because of surrounding environment. Based on Paul Ekman in 20th century, he identified six basic emotions which are anger, disgust, fear, happy, sad and surprise [6]. Human emotion usually changes based on its surrounding and different actions will be taken in different situation. One of the most important components of the modern tertiary education system in academic institutions is student's emotional states 
towards their lecturer [7]. Therefore, an understanding of the nature of emotions, its eliciting conditions, and emotional experienced within the academic environment and setting context are important [8]. Human researcher throughout the world has been trying to find image processing evolution step especially in human face detection [9].

In recent years, researches have made considerable progress in developing facial expression classifier. There are some works relate with facial expression or emotion. A facial expression recognition system has been developed to identify the facial image and automate face detection performed by using the face detector [10]. However, it is lack in term of performance where more classifier is suggested as future work. Another related work is automated facial emotion analysis system for students in classroom environment [11]. This work is to analyze whether the emotion of the students with respect to comprehension are indicated through expressions of facial action units which are eyes, mouth, eyebrows and forehead. However, it is this research unable to detect images from different angle. Convolutional neural network $(\mathrm{CNN})$ for facial expression recognition task has been developed.

Various CNNs for a facial expression recognition problem are developed such as shallow model and deep model. The output was analyzed using various post-processing and visualization techniques. The images of faces are used and Histogram of Oriented Gradient (HOG) feature is used for facial expression recognition because they are sensitive to edge. However, this research not able to detect images with multiple color [12]. Therefore, a research on student emotion estimation based on facial application in E-Learning during has been develop where this research focusing on COVID-19 pandemic situation.

\section{FACIAL APPLICATION FRAMEWORK}

Framework in Figure 1 started with image acquisition followed by pre-processing image using grayscale conversion and contrast stretching. Face detection using Haar Cascade method with face model for identifying the localization of eyes and mouth region. Skin color segmentation for segment the image of eyes and mouth region. Feature extraction using Grey-Level Co-Occurrences Matrix (GLCM). Feature classification using Support Vector Regression (SVR) technique, testing and result.

\subsection{Image Acquisition}

Image acquisition is the first step of image processing. In this phase, the data will be collected. All data were collected from online database. There are four different images of human face will be collected and use in this research. It consists of four (4) types of emotion which surprise, happy, sad or normal. The images of human face must be in size $640 \times 490$ pixels to get the better result for emotions classification. Thirty (30) face images consist of different kind of emotions from Extended Cohn Kanade Image Database [13] had been collected to be used in training data. All the images used in this project are in grayscale format.

\subsection{Pre-Image Processing}

In this stage, first, the image needs to be converted into grayscale that only consist shades from black to white colour in the image. Then, face image is going through contrast stretching technique using histogram equalization will be applied to the image. This will enhance image background color and saturation.

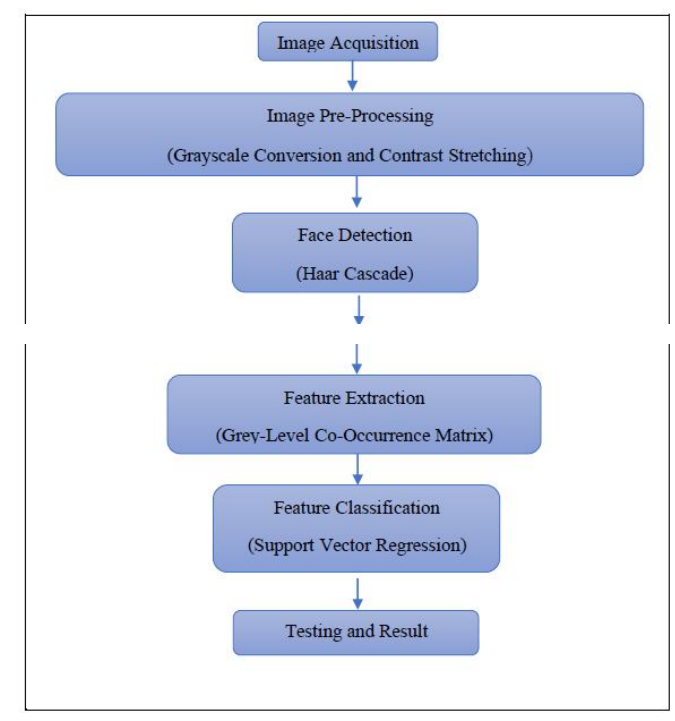

Figure 1: Facial application framework

\section{Contrast Stretching using Histogram Equalization}

Firstly, the face image needs to be converted into grayscale format which only consist the shades of black and white. Then, the face image will go through contrast stretching by increase the dynamic range of histogram is technique of histogram equalization which can improve contrast. The histogram equalization goal is to acquire a consistent histogram. New intensity value assign in each pixel based on its earlier intensity level [14]. The histogram equalization start with histogram formation where the color of image data is collected and represent in the histogram. Next, calculate new intensity values for each intensity levels using equation in (1). Emotions are a key component of effective learning and problem-solving, particularly when it comes to engaging with computer-based learning environments [5]. Max. Intensity Level is the maximum intensity level which a pixel can get. Expression in the bracket is number of pixels that have intensity below or equal to output intensity level. $O i$ represent the new intensity value where $i$ is a previous 
intensity level. Finally, previous intensity values are replaced with the new intensity values. Figure 2 shows the implementation of contrast stretching on the sample dataset.

$$
o_{t}=\left[\sum_{f}^{i} N_{f}\right] \times \begin{gathered}
\text { Maxi Intensity Level } \\
\text { No. of Pixels }
\end{gathered}
$$

\subsection{Face Detection}

The existence of features in an image will be detected by Haar features. Increasing function produces a single value obtained by subtracting the number of pixels in a white rectangle from the number of pixels in a black rectangle [15]. Haar-like features work by scanning the image starting from upper left corner until lower right corner of the image. The scanning process is done a few times for detecting the human face. The concept of basic image is used for calculating the features in rectangular form in faster way. For the calculation the number

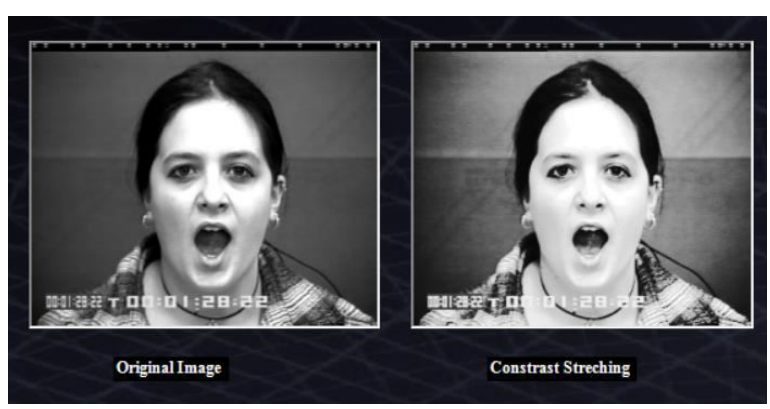

Figure 2: Contrast Stretching technique on the image

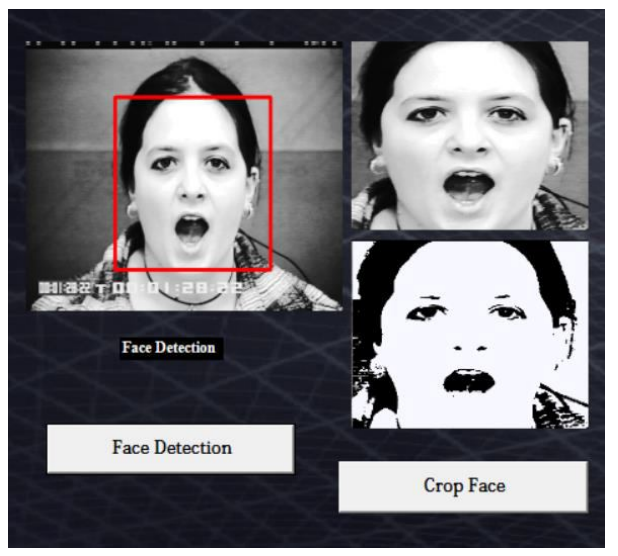

Figure 3: Face area detection using Haar features

of pixels in given rectangle, four values are needed for each rectangular corner. Pixels value $(x, y)$ in the basic image is the number of above pixels and on the left $(x, y)$. Figure 3 shows the implementation of Haar features where the area region of the face is detected and highlighted with a red box. Then, the detected area is cropped and divided into two segments. One is grayscale image and another one is binary image which consist of black and white color only. The purpose of this process is to make the cropping region of the eyes and mouth region more accurate. It can be achieved by highlighting the important area to be analyzed and ignore the other area.

\subsection{Eye and Mouth Detection}

The area of eyes and mouth are cropped using face model technique. Face model will calculate the upper right, upper left and bottom center of the region that want to be cropped. For the eye's detection, the face width will be calculated. Then, Find the middle location of both eyes. The calculation of range for middle position is shown in (2):

$$
\text { middle position }=\frac{\text { width }}{4} \ldots\left(\text { width }-\frac{\text { width }}{4}\right)
$$

Along the height of the scale, the highest white continuous pixel is the middle location of the two eyes. Then, search the vertical for finding the starting upper position for two eyebrows. The calculation for finding the range for left eye is shown in (3) and the range for right eye is shown in (4). Assume the middle position value is mid.

$$
\begin{aligned}
& \text { right eye position }=\text { mid } \ldots . \text { width }-\frac{\text { width }}{8} \\
& \quad \text { left eye position }=\frac{\text { width }}{8} \ldots \text { mid }
\end{aligned}
$$

Next, place the continuous black pixels vertically from eyebrow to the eye to make the eyebrow and eye connected. The calculation for the range between for left eye vertical black pixel-lines place is shown in (5) and right eye (6). Assume that black pixel-lines range value is denoted by $b p$ left and $b p$ right.

bp left $=\frac{m i d}{2} \ldots \frac{m i d}{4}$

$b p r i g h t-m i d+\frac{(\text { width }- \text { mid })}{4} \ldots$. mid $+3 x \frac{(\text { width }- \text { mid })}{4}$

Then, search black pixel vertically to find the lower position of the two eyes. The calculation to find the range of black pixel vertically for right eye is same as (7).

bp left $=\frac{\text { mid }}{4} \ldots . . m i d-\left(\frac{\text { mid }}{4 \times \text { width }}\right)$

Next, find the black pixel horizontally from middle position to the starting position of black pixels for finding the right side of the left eye between the lower and upper position of left 
eye. For the left side of the eye, search the middle position to the starting position of black pixels between the lower and upper position of right eye. The starting width of the image is the left side of the left eye and the ending width of the image is the right side of the right eye. Lastly, cut the upper position, lower position, left side and right side of the two eyes from the image.

For the region of the mouth, first, determine the distance between forehead and eyes. The value of position of the right eye and left eye calculated based on (5) and (6) are used to determine the box area of the mouth. In the case of the mouth region, the starting point is at $1 / 4$ of the left eye box and the end point is at $3 / 4$ of the right eye box. Figure 4 shows the result of eyes and mouth region box that being cropped from

\subsection{Feature Classification}

In classification stage, Support Vector Machine (SVM) and Support Vector Regression (SVR) are used for emotion classification. SVM is the best method in image and pattern classification. SVM is a prediction and classification tool that use theory of machine learning. This is to predict accuracy in minimize way and avoid over-fit to the data automatically. It is a system that use hypothesis space of a linear functions in a high dimensional feature space that trained with a learning algorithm from theory of optimization [16].

SVM are the maximal hyperplane classification method which depends on statistical learning theory results to ensure the high performance of generalization. Some basic theory of

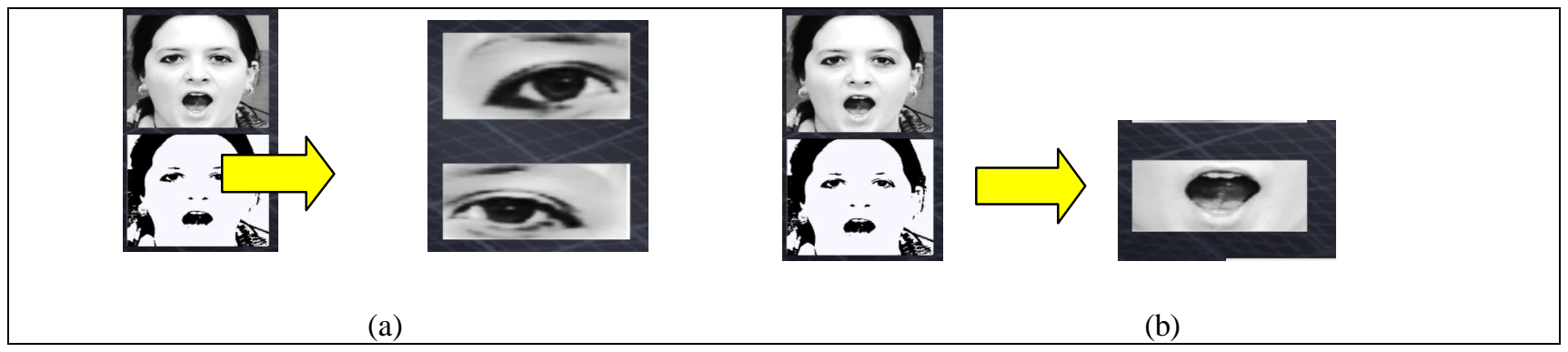

Figure 4: Eyes (a) and mouth (b) region box that being cropped from the face area

the face area.

\subsection{Feature Extraction}

The black and white image of eyes and mouth regions are trained using Grey-Level Co-Occurrence Matrix (GLCM) for texture analysis. The value of contrast, energy and homogeneity are calculated from GLCM for training data is shown in Figure 5.

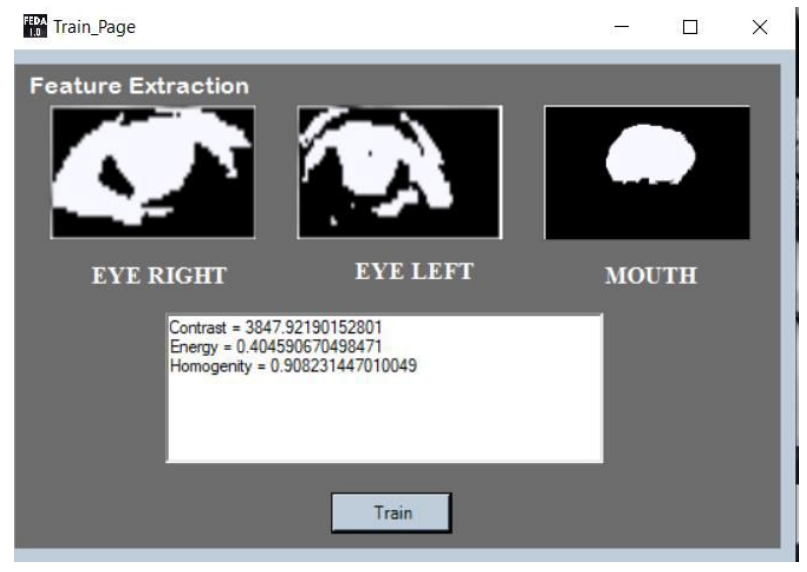

Figure 5: GLCM features extraction from mouth and eyes images two-class classification problem of SVM. This to achieve isolate two classes by a function which is generate from available example. There are many possible linear classifiers which can separate data. However, there is only one which maximizes the margin. Margin are shown in figure 6.

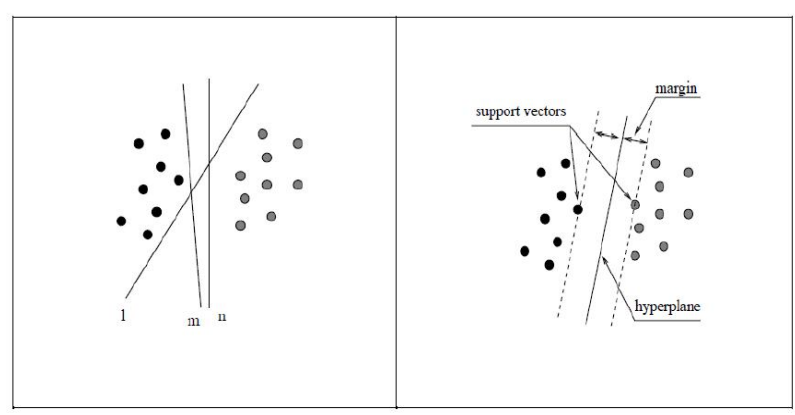

(a)

(b)

Figure 6: a) $1, \mathrm{~m}$ and $\mathrm{n}$ are arbitrary hyperplanes b) The separating hyperplane with the largest margin [17]

The optimal separating hyperplane (OSH) is called for this linear classifier [18]. Naturally, we might expect this boundary to generalize well from the other possible boundaries shown in Figure 7. 
Nurbaity Sabri et al., International Journal of Advanced Trends in Computer Science and Engineering, 9(1.4), 2020,576 - 582

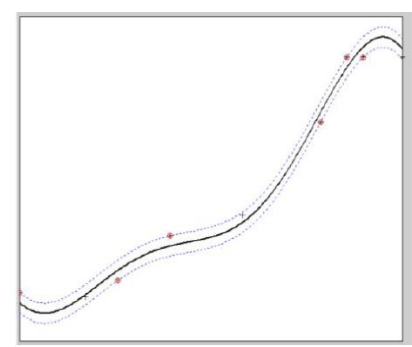

Figure 7: SVR using RBF kernel [18]

Classification is the process of predict and classify image into different type of emotions. It also gives accuracy percentage of the prediction to the user. Support Vector Regression (SVR) is used to predict the value of the emotion label and the name of emotion is classified from the range of predicted value. The dataset consists of 30 sample of different person's frontal image with four (4) types of emotion which are happy, normal, surprise and sad. The total image in the dataset is 120. The range of value which more than or equal to 0.0 until value less than 1.0 will be predicted as happy emotion. Next, the range value which more than or equal to 1.0 until value less than 1.5 will be predicted as normal emotion. The range value more than or equal to 1.5 until value less than 2.0 will be predicted as sad emotion. Lastly, the range value more than or equal 2.0 until less than 3.0 will be predicted as surprise emotion.

\section{EXPERIMENTAL RESULT}

The total data in the dataset is 120 images. It consists of 30 different person's frontal face image. Each person has 4 different images of different face expression that show their emotion which are happy, normal, surprise and sad emotion. All of these images are tested using Support Vector Regression (SVR) and Support Vector Machine (SVM). The framework used is EncogSVM. After the testing is done, all the results of testing will be summarized using a confusion matrix for calculating the accuracy of the prediction and classification. The testing result of the emotion type classification is divided into four which are happy, normal, surprise and sad. Thirty (30) images for each type of emotion are used for testing and analysis. The images that use in training are also use in testing phase. Table 1 shows the result of the emotion classification after testing using Support Vector Regression (SVR) model and Support Vector Machine (SVM).
Table 1: Classification Result using SVR and SVM

\begin{tabular}{|l|l|l|l|l|}
\hline $\begin{array}{l}\text { Machine } \\
\text { Learnin } \\
\text { g Model }\end{array}$ & $\begin{array}{l}\text { Emotion } \\
\text { Type }\end{array}$ & $\begin{array}{l}\text { Number } \\
\text { of } \\
\text { Tested } \\
\text { Image }\end{array}$ & $\begin{array}{l}\text { Number } \\
\text { of Image } \\
\text { Identified } \\
\text { Correctly }\end{array}$ & $\begin{array}{l}\text { Number of } \\
\text { Image } \\
\text { Identified } \\
\text { Incorrectly }\end{array}$ \\
\hline SVR & Happy & 30 & 0 & 0 \\
\cline { 2 - 5 } & Normal & 0 & 30 & 0 \\
\cline { 2 - 5 } & Sad & 0 & 0 & 29 \\
\cline { 2 - 5 } & Surprise & 0 & 0 & 0 \\
\hline \multirow{5}{*}{ SVM } & Happy & 20 & 6 & 0 \\
\cline { 2 - 5 } & Normal & 17 & 7 & 2 \\
\cline { 2 - 5 } & Sad & 16 & 6 & 2 \\
\cline { 2 - 5 } & Surprise & 14 & 3 & 2 \\
\hline
\end{tabular}

The entire image tested for happy emotion is correct for SVR classifier. Followed by sad and surprise emotion. However, there is only 1 image is identified incorrectly for sad emotion type. Meanwhile for SVM, only 20 images are identified correctly for happy emotion while another 10 is incorrect. For the normal emotion, the number of identify correctly is 7 and 23 images are identified incorrectly. Only 2 images are identified correctly for sad emotion and other 28 are incorrectly identified. The number of images for surprise emotion type that identified correctly is 11 and another 19 images are identified incorrectly. Table 2 shows accuracy result being calculated by confusion matrix for face emotion type classification. The overall accuracy percentage is calculated by the formula in (8).

Accurnoy $\%=\frac{\text { Total TRUE value }}{\text { Tolal images lesting }} \times 100$

From the result, the highest accuracy obtained is $99.16 \%$ by using Support Vector Regression (SVR) while the result obtains using Multiclass Support Vector Machine (SVM) is 33.33\%. Support Vector Regression and Support Vector Machine share the same goals which are to find a hyperplane in the feature space. However, SVM only able to find hyperplane that breaks up data into two parts, while SVR can find a hyperplane that accurately predict distribution of 
Nurbaity Sabri et al., International Journal of Advanced Trends in Computer Science and Engineering, 9(1.4), 2020,576 - 582

original data [19]. Therefore, SVR is more accurate to predict and classify the type of human emotion based on facial image. This shows that the application to identify the type of emotion from face image using SVR is acceptably to be used and proven that this research achieves its objective.

Table 2: Accuracy Result using SVR and SVM

\begin{tabular}{|c|c|c|c|}
\hline Model & $\begin{array}{c}\text { Total } \\
\text { images } \\
\text { testing }\end{array}$ & $\begin{array}{c}\text { Total } \\
\text { TRUE } \\
\text { value }\end{array}$ & $\begin{array}{c}\text { Accuracy } \\
(\%)\end{array}$ \\
\hline SVR & 120 & 119 & $99.16 \%$ \\
\hline $\begin{array}{c}\text { SVM } \\
\text { Multiclass }\end{array}$ & 120 & 40 & $33.33 \%$ \\
\hline
\end{tabular}

\section{CONCLUSION}

This study focuses on the estimation of student emotion based on facial application in e-learning during the COVID-19 pandemic. This research aims to improve current e-learning method by identify emotion recognition of student on e-learning during pandemic while linking emotion detection to adapted learning activities. The classification accuracy using SVR shows promising result on identify four (4) types of face emotion which is happy, normal, surprise and sad. Further development of other human recognition, such as speech, will be done in the future to enhance online e-learning, especially during the COVID-19 pandemic.

\section{ACKNOWLEDGEMENT}

This research was supported by Universiti Teknologi MARA, through the Skim Geran Dalaman Teja 2020 GDT2020-36.

\section{REFERENCES}

1. M. A. Almaiah, A. Al-Khasawneh, and A. Althunibat. Exploring the critical challenges and factors influencing the E-learning system usage during COVID-19 pandemic, Education and Information Technologies, vol. 1, 2020.

2. M. P. Murphy. COVID-19 and emergency eLearning: consequences of the securitization of higher education for post-pandemic pedagogy, Contemporary Security Policy, pp. 1-14, 2020.

3. R. Xu, J. Chen, J. Han, L. Tan, and L. Xu. Towards emotion-sensitive learning cognitive state analysis of big data in education: deep learning-based facial expression analysis using ordinal information, Computing, pp. 1-16, 2019.

4. V. Bradáč, and P. Smolka. Personalised English language education through an e-learning platform, in Asian Conference on Intelligent Information and Database Systems, Singapore, pp.517-526, Springer, Singapore, March 2020.

5. M. Magdin, M. Turčáni, and L. Hudec. Evaluating the emotional state of a user using a webcam, International Journal of Interactive Multimedia \& Artificial Intelligence, vol. 4, 2016. https://doi.org/10.9781/ijimai.2016.4112

6. P. Ekman, and G. Yamey. Emotions revealed: recognising facial expressions: in the first of two articles on how recognising faces and feelings can help you communicate, Paul Ekman discusses how recognizing emotions can benefit you in your professional life, Student BMJ, vol. 12, pp.140-142, 2004.

7. M. Zaliman, M. Yusoff, N. A. Hamid, M. A. Rahman, and N. H. Jaafar. Can students' emotional states influence their assessment of lecturers' performance?, vol. 4, pp. 96-105, 2012.

8. F. D. Ahmed, A. Y. C. Tang, and M. S. Ahmad. Recognizing student emotions using an agent-based emotion engine, International Journal of Asian Social Science, vol. 3, pp. 1897-1905, 2013.

9. K. Dewoolkar, G. Bhole, A. Mehta, and A. Choudhari. Facial expression recognition using image processing, 2014.

10. N. Matang, S. Sunuwar, S. Shrestha, and S. Parajuli. A facial expression recognition system, 2016.

11. S. Sharmila, A. Kalaivani, \& S. Gr. Automatic facial emotion analysis system for students in classroom environment, International Journal of Pure and Applied Mathematics, vol. 119, pp. 2887-2894, 2018.

12. S. Alizadeh, and A. Fazel. Convolutional neural networks for facial expression recognition, pp. 1-6, 2014.

13. L. Patrick, J. F. Cohn, T. Kanade, J. Saragih, and Z. Ambadar. The extended Cohn-Kanade dataset (CK+): A complete dataset for action unit and emotion-specified expression, CVPR for Human Communicative Behavior Analysis, pp. 1-8, 2010.

14. S. S. Bagade, and S. K. Vijaya. Use of histogram equalization in image processing for image enhancement, International Journal of Software Engineering Research \& Practices, vol. 1, pp. 6-10, 2010.

15. A. Priadana, and M. Habibi. Face detection using Haar Cascades to filter selfie face image on Instagram, International Conference of Artificial Intelligence and Information Technology (ICAIIT), pp. 6-9, 2019.

16. E. Andana, M. Othman and R. Ibrahim. Comparative analysis of text classification using Naive Bayes and Support Vector Machine in detecting negative content in Indonesian twitter, International Journal of Advanced Trends in Computer Science and Engineering, vol. 8, 2019.

https://doi.org/10.30534/ijatcse/2019/6481.32019 
Nurbaity Sabri et al., International Journal of Advanced Trends in Computer Science and Engineering, 9(1.4), 2020,576 - 582

17. N. Mohd Esa, A. Mohd Zain and M. Bahari. A Grey wolf optimizer based Support Vector Machine for classification of finger movement finger movement, International Journal of Advanced Trends in Computer Science and Engineering, vol. 8, 2019.

https://doi.org/10.30534/ijatcse/2019/1981.62019

18. Y. Reddy and Y. Prasanth. A Hybrid Quartile deviation-based Support vector regression model for software reliability datasets, International Journal of Advanced Trends in Computer Science and Engineering, vol. 9, 2020.

https://doi.org/10.30534/ijatcse/2020/30922020

19. C. J. Wang, and C.Y. Chang. Based on Support Vector Regression for emotion recognition using physiological signals, International Joint Conference on Neural Networks, pp. 1-8, 2010. 